\title{
Binocular interaction in induced line rotation
}

\author{
MICHAEL T. SWANSTON \\ Dundee College of Technology, Dundee, Scotland \\ and \\ NICHOLAS J. WADE \\ University of Dundee, Dundee, Scotland
}

\begin{abstract}
Binocular interaction in induced movement was studied using the apparent rotation of a line superimposed on an expanding or contracting grating. The symmetrical transformation reduced the probability of differently directed tracking eye movements' influencing the outcome with dichoptic stimuli. Presentation of the line and background to separate eyes eliminated the apparent rotation, but this was likely to have been due to binocular rivalry. When the inducing field was itself split into dichoptically presented components, an induced rotation was obtained of around half the monocular value. However, the dichoptic rotation could be accounted for by the effects of the two monocular half-fields, when presented in isolation. If backgrounds of differing spatial properties were seen by each eye, the combined effects were predictable from their independently measured monocular effects. It is concluded that the results provide little support for the occurrence of dichoptic induced line rotation.
\end{abstract}

Induced movement occurs when relative motions in the visual field appear to be distributed among the moving and static objects differently from their actual displacements. Thus a stationary point may seem to be moving with respect to a larger moving frame (Duncker, 1929; Ehrenstein, 1925), or the perceived direction of a moving point may appear displaced when seen in conjunction with objects moving in other directions (Gogel \& Griffin, 1982; Wallach, Bacon, \& Schulman, 1978). The distribution of apparent movement is likely to be influenced by all the factors that displace stimuli on the retina over time. For example, in a Duncker-type dot-and-frame display, the eyes could follow the moving frame in order to yield the oppositely directed retinal motion of the stationary dot (see Kaufman, 1974). Considerable evidence has been marshaled to refute this interpretation (Bassili \& Farber, 1977; Brosgole, Cristal, \& Carpenter, 1968; Schulman, 1979), and theories of induced movement tend to be expressed in terms of the effectiveness of the cues for relative motion. Thus, factors like enclosure (Wallach, 1959) or adjacency (Gogel \& Koslow, 1972) tend to make one moving pattern the frame of reference for the other, irrespective of the physical motions.

According to such frame-of-reference theories, the magnitude of induced movement should not be reduced under conditions of dichoptic observation, provided that the

This research was sponsored by the Medical Research Council of Great Britain (Project Grant G8104633N). The assistance of Georgina Swanston with the collection of data is gratefully acknowledged.

M. T. Swanston's mailing address is: Dundee College of Technology, Bell Street, Dundee DD1 1HG, Scotland. N. J. Wade's mailing address is: Department of Psychology, University of Dundee, Dundee DDI 4HN, Scotland. scene is phenomenally equivalent to that observed under monocular or binocular conditions. However, if induced movement is in part the result of simultaneous motion contrast (Anstis \& Reinhardt-Rutland, 1976), then this would not necessarily be the case. Processes in the visual system that are sensitive to relative motion may be partly or wholly monocular, and could thus give rise to less induced movement with dichoptic viewing. The evidence concerning binocular interaction in induced movement is relatively limited, and not consistent in the reported findings. This may in part be due to differences in the types of induced movement displays employed. For example, Day and Dickinson (1977) found that lateral motion of a central grating surrounded by a larger grating could be induced dichoptically when the whole surround was displaced laterally, but not when the surround motion was within a stationary boundary (see, also, Over \& Lovegrove, 1973). It is also possible that a stationary boundary would reduce any pursuit eye movements that were likely to have occurred in the former condition. Levi and Schor (1984) monitored eye movements during dichoptic observation of induced linear motion, and limited analysis to judgments made when no pursuit movements occurred. In these cases, the induced movement was said to be "weak, " but "present." It is clear that eye movements add to the difficulty of examining induced movement dichoptically.

The experiments reported here employed a type of induced movement in which the inducing field consisted of a grating transformed by a uniform optical expansion or contraction. This generates radial motion away from or towards a central stationary point and is therefore unlikely to give rise to tracking eye movements. An oblique line 
superimposed on such an expanding grating field undergoes an induced rotation, due to a perceived relative motion between the line and the background.

Since the movement of the background field is perceived only in the direction at $90^{\circ}$ to its orientation, as in the "barber-pole" effect described by Wallach (1959), there is a relative movement between the line and the background that is in an opposite direction in each half of the field. The magnitude of this relative motion increases with distance from the focus of expansion, and thus yields a perceived rotation of the line (Swanston \& Wade, 1981; Wade \& Swanston, 1984b). This phenomenon provides a means of testing for dichoptic induced movement which reduces the conflicting stimuli for pursuit eye movements associated with other configurations. The symmetrical motion in each half of the visual field ensures that there is no systematic bias in the direction of any tracking eye movements. Although, in principle, any given line in the background field could be fixated and tracked, this would give rise to an unidirectional lateral motion of the static test line rather than a rotation. In addition, it appears that such a uniform radial expansion acts in practice as an effective stimulus for fixation of the stationary central portion.

\section{EXPERIMENT 1}

The simplest approach to measuring dichoptic induced movement is to present the inducing field and induced target to separate eyes. This experiment was therefore designed to compare the induced rotation described above with monocular and dichoptic observation. That is, an oblique line and a vertical grating were presented either to the same eye or to different eyes.

\section{Method}

Apparatus. The apparatus was based upon that described by Wade and Swanston (1984a), extended to provide two independent video channels (see Figure 1). Two video cameras (Sony AVC $3250 \mathrm{CE}$ ), each with a motorized zoom lens (Fujinon H $5 \times 14$ MA1) were linked via a signal mixer (Viscount 1107) to monochrome video monitors (Hitachi VM $900 \mathrm{E} / \mathrm{K}$ ) situated in a darkened room. Each monitor was covered by a mask with a circular aperture, which subtended $6^{\circ}$ at the subject's eyes for the viewing distance of $114 \mathrm{~cm}$. The monitors were seen through a pair of biprisms which could be adjusted to bring circular apertures into alignment.

The background field consisted of a vertical square-wave grating mounted on a rotatable shaft, with its center aligned with the optical axis of a zoom lens. The target line was inclined at $45^{\circ}$ to the grating, since this was found to produce the maximum perceived rotation (Swanston \& Wade, 1981). It could be attached either to the grating or to the surface of a blank disk (see Figure 2). The line was long enough for the ends not to be visible to the subject at any zoom setting. The mixer allowed the experimenter to change the field presented to each eye.

Trials consisted of a 2 -sec period of zoom-in, during which the spatial frequency of the background decreased from 5.3 to $2.2 \mathrm{cy}$ cles/deg. The line simultaneously changed in width from $8 \mathrm{~mm}$ $\left(0.4^{\circ}\right)$ to $20 \mathrm{~mm}\left(1.0^{\circ}\right)$.

Subjects. Twelve subjects took part in the experiment. All had normal or corrected-to-normal vision.

Procedure. The four stimulus configurations are shown in Figure 2. The subjects viewed each condition 10 times, in a random order. Following the 2 -sec trial, the subjects reported the extent of the apparent rotation of the oblique line in degrees signed positively for clockwise and negatively for counterclockwise rotation. Previous studies of the effect have shown that this reporting

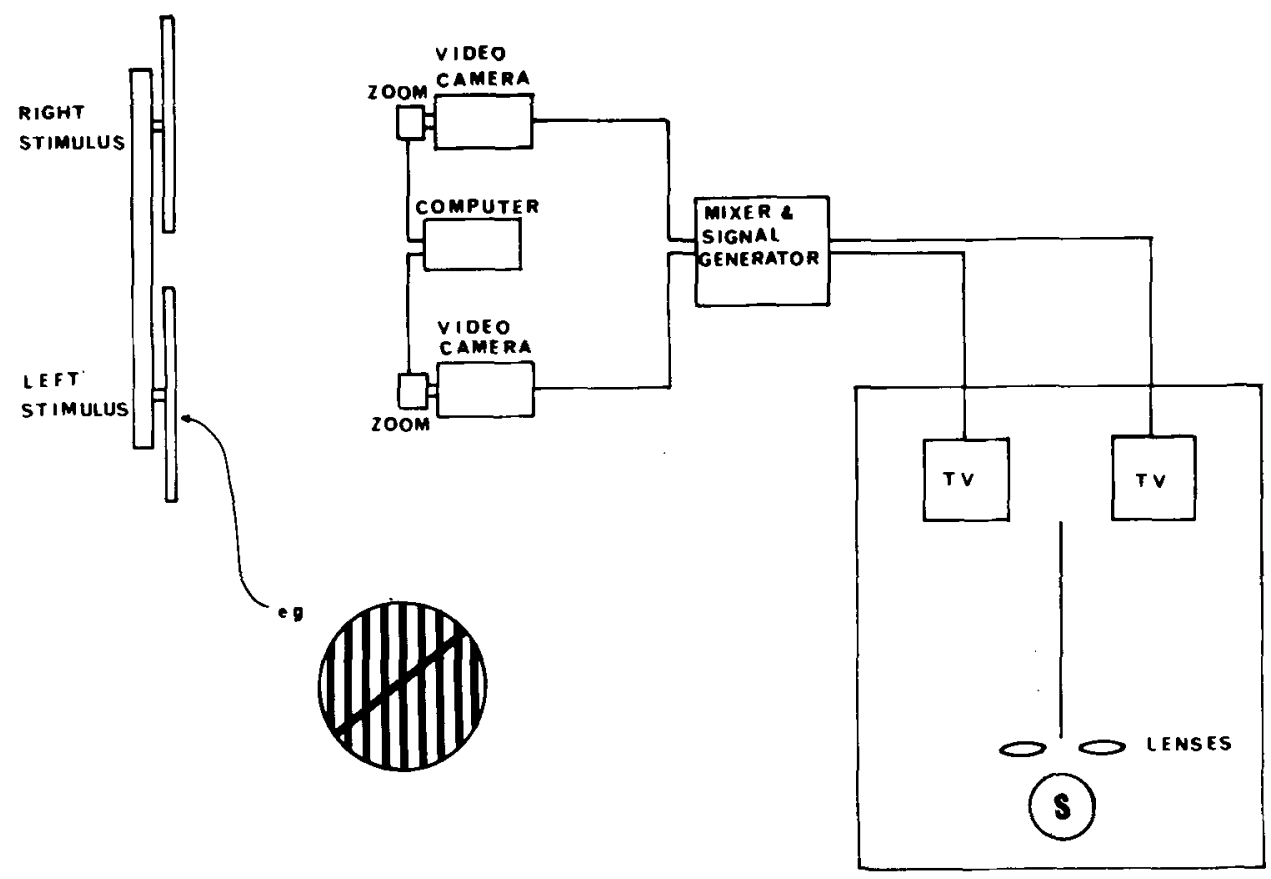

Figure 1. The apparatus used in Experiment 1. In the example shown, the oblique line and grating were presented to the left eye and a gray field was presented to the right. 


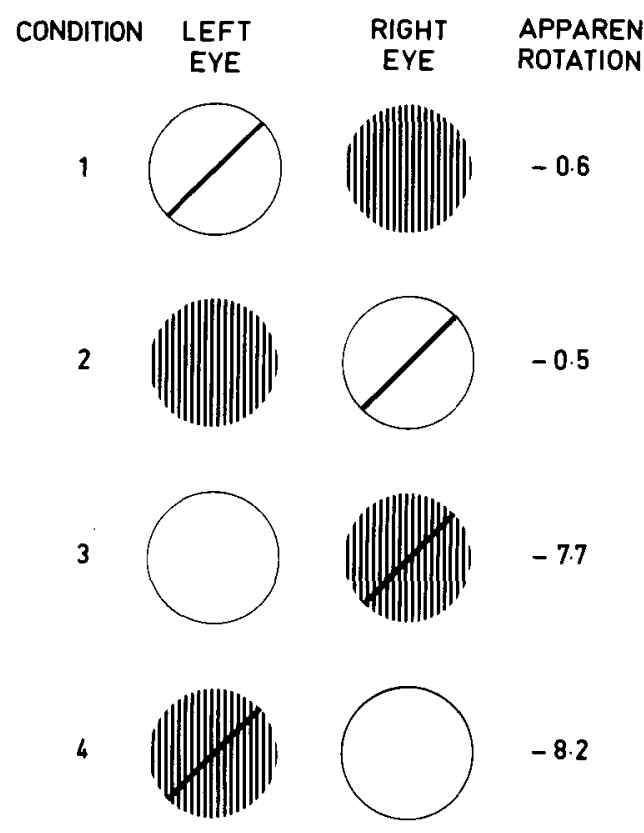

Figure 2. Stimulus displays for dichoptic and monocular presentation in Experiment 1. The displays represent schematically the initial stimulus dimensions, which were transformed during a 2 -sec zoom-in. The mean degrees of apparent rotation are given for each condition: Negative values denote counterclockwise apparent rotation.

procedure is reliable and easily followed by naive subjects (e.g., Swanston \& Wade, 1981; Wade, Swanston, Ono, \& Wenderoth, 1983).

\section{Results}

Mean perceived rotation scores were derived for each condition; these are shown in Figure 2. Both monocular conditions produced perceived rotations of around $8^{\circ}$, which corresponds to values found in earlier studies. However, the dichoptic conditions showed no evidence of induced movement in the line.

A possible explanation of this outcome is that the dichoptic display was ineffective because of rivalry between the background and the oblique line. This would have suppressed the region around the intersections between the line and background, which was likely to have been the strongest source of induced movement due to adjacency. It should be noted that rivalry suppression need not necessarily prevent perceptual interactions; for example, it is known that the tilt illusion persists even when the tilted background field is invisible due to rivalry (Wade, 1980). Alternatively, it could be that, regardless of rivalry, induced movement does not occur between dichoptically presented stimuli.

\section{EXPERIMENT 2}

The rivalry between the line and the background in Experiment 1 prevented any clear conclusion regarding the extent of binocular interaction in induced movement. In order to address this issue more directly, the display was split into the component half-fields, and these were presented either monocularly or dichoptically. That is, a line was always present, but the gratings above or below it were generated independently and the eye or eyes to which they were presented were varied systematically (see Figure 3). The simplest comparison would be between monocular and dichoptic observation of an oblique line with a vertical background (Conditions 1 and 5, respectively), because these displays would be phenomenally equivalent. More complex combinations of the half-fields can be presented with this arrangement, however-ones that are potentially informative about the interaction of movement fields within a phenomenally single display. For example, in Conditions 2 and 6, zoom-in on the upper half (A) would be expected to yield a counterclockwise apparent rotation of the line, as would zoom-out on a horizontal grating (C). By contrast, zoom-in on the vertical upper half-field $(A)$ and on the horizontal lower field (B) should give opposing directions of apparent rotation; this combination is represented by Conditions 3 and 7 . The same approach applies to Conditions 4 and 8 , with zoom-in on a vertical upper field (A) and zoom-out on a vertical lower field (D). The final four conditions (912) examined the effects of the monocular half-fields in isolation.

\section{Method}

Apparatus. The apparatus was essentially the same as that used for Experiment 1. However, an additional mixer and video camera

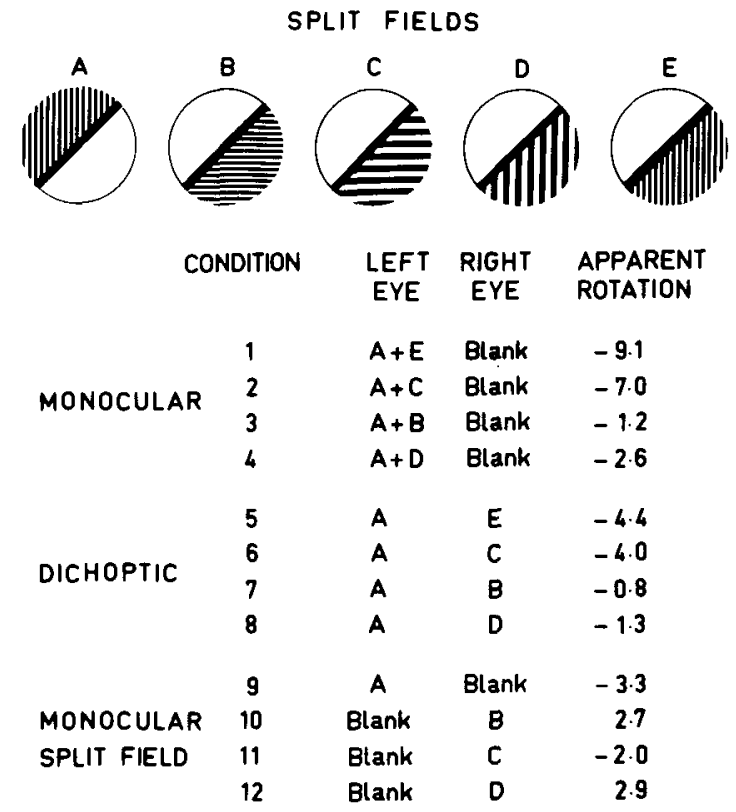

Figure 3. The split fields employed in Experiment 2 are shown schematically. The dimensions represent the initial states before zooming: $A, B$, and $E$ were transformed by zoom-in, whereas $C$ and $D$ received zoom-out. The combinations of the split fields in the monocular and dichoptic conditions and the mean degrees of apparent rotation (negative values denote counterclockwise rotation) are given. 
were used to create and manipulate the split fields required. A third camera was placed in front of a black screen on which was attached a white line at $45^{\circ}$. The output of this camera was used as a keying input to the two mixers, and thus formed a static black line unaffected by other manipulations. Each of the background fields was covered obliquely by a white mask, so that one showed the upper part of a square-wave grating and the other, the lower. The appearance of these fields is illustrated in Figure 3. The orientation of one field could be set as required, and the signals could be switched between the subject's monitors by the experimenter. The oblique line did not change in width during zooming.

Subjects. Twelve subjects took part in the experiment. All had normal or corrected-to-normal vision.

Procedure. There were 12 experimental conditions, which are best described in three groups: monocular whole field (Conditions 1-4), dichoptic whole field (Conditions 5-8), and monocular split field (Conditions 9-12). The monocular whole-field conditions were displayed on the left monitor, the other showing a gray field. In each case, the upper left half-field contained a vertical grating like that used in the earlier experiments, which was always zoomed in. The resulting dimensional changes of the background corresponded to those in Experiment 1. The lower half-field contained a grating that was either vertical or horizontal; these were seen both with zoom-in and zoom-out. The dichoptic conditions consisted of the same half-fields, but were displayed separately on the left (upper field) and right (lower field) monitors. In the monocular split-field case, the component half-fields described above were seen independently on separate trials. The subject's task was to estimate, in degrees, the extent and direction of any apparent rotation of the oblique line.

\section{Results}

The mean perceived rotations of the oblique line are shown in Figure 3, for each of the 12 conditions. Display $\mathrm{E}$ was not presented alone, but its value was assumed to be the same as that for display $\mathrm{A}$, which was the equivalent upper split field. Zoom-in on a vertical grating produced counterclockwise line rotation under both monocular and dichoptic observation (Conditions 1 and 5 , respectively). A similar pattern of results was found for Conditions 2 and 6 , in which the two half-fields differed in orientation and zoom phase, but cooperated in inducing counterclockwise line rotations. Thus, despite the differences of appearance of the two movement halffields, they were effective in combination. However, the extent of induced line rotation was significantly greater with monocular viewing $[\mathrm{F}(1,121)=10.31, \mathrm{p}<.001]$. When the half-fields gave opposite directions of the line rotation (Conditions 3, 4, 7, and 8), the apparent rotation tended towards zero. The residual counterclockwise rotations that were reported correspond to an earlier finding that larger apparent rotations are induced by zoomout with a vertical background (Wade, Swanston, Ono, \& Wenderoth, 1983).

This evidence would appear to favor an interpretation in terms of binocular interaction: The dichoptic effects were around $50 \%$ of those obtained monocularly, and this corresponds quite well to the values measured for the interocular transfer of spatial aftereffects (Moulden, 1980) and for dichoptic presentation of orientation illusions (Wade, 1980). These have been interpreted as evidence for discrete populations of visual cortical cells responsive to earlier monocular or binocular stimulation. However, this conclusion is made less secure by the results of the monocular split-field conditions (9-12). It is clear that these do induce line rotations in the directions expected from whole-field stimulation, and that their combinations could account for the dichoptic effects. This is supported statistically, since in no case did the dichoptic effect differ significantly $(p>.05)$ from the sum of the corresponding half-field combinations.

As noted above, Levi and Schor (1984) did find a small degree of induced movement during dichoptic observation, although no quantitative values were given to allow its comparison with the monocular effects.

While the results of the present experiment do not provide any support for binocular interaction in induced line rotation, they do not preclude the operation of binocular processes. If induced rotation can be generated by stimuli presented to each eye independently and then summed at the level of binocular interaction, this would account for the results (cf. Anstis \& Duncan, 1983). Identification of a purely binocular process would require stimuli that produce no induced movement when seen monocularly, but this was not the case in the configurations employed here.

\section{EXPERIMENT 3}

An alternative method of investigating binocular integration is to use dichoptic displays in which both fields generate an induced rotation when viewed monocularly. The target line is present in each eye, but superimposed on backgrounds that generate independent perceived rotations that can be varied in either an opposing or a complementary manner. In principle, the resultant perceived rotation should reflect any binocular interaction between the two movement fields.

\section{Method}

Apparatus. The apparatus was esssentially the same as that used in Experiment 1. The oblique line was generated electronically and superimposed on the background fields by the mixer. This permitted the use of two background gratings, and ensured that the line was identical for both. As in Experiment 2, the line did not undergo any change of dimensions during zooming, but this was found from observation to have no detectable effect on the perceived rotation. When zoom-out was employed, this produced a background transformation opposite to that given by zoom-in.

Subjects. Eighteen subjects, all with normal or corrected-tonormal vision, took part in the experiment.

Procedure. The seven experimental configurations are shown in Figure 4. The subjects viewed each condition five times and, as in Experiment 1, reported any perceived rotation of the line in degrees. Conditions 1-3 were monocular controls, differing in the direction of zooming or the orientation of the background. In Conditions 4-7, each eye saw a movement field, each of which could independently produce a perceived rotation in either the same or opposed directions. This was achieved by appropriate combinations of zoom direction and background orientation.

\section{Results}

The mean rotation scores are shown in Figure 4, alongside the corresponding display conditions. Each of the first 


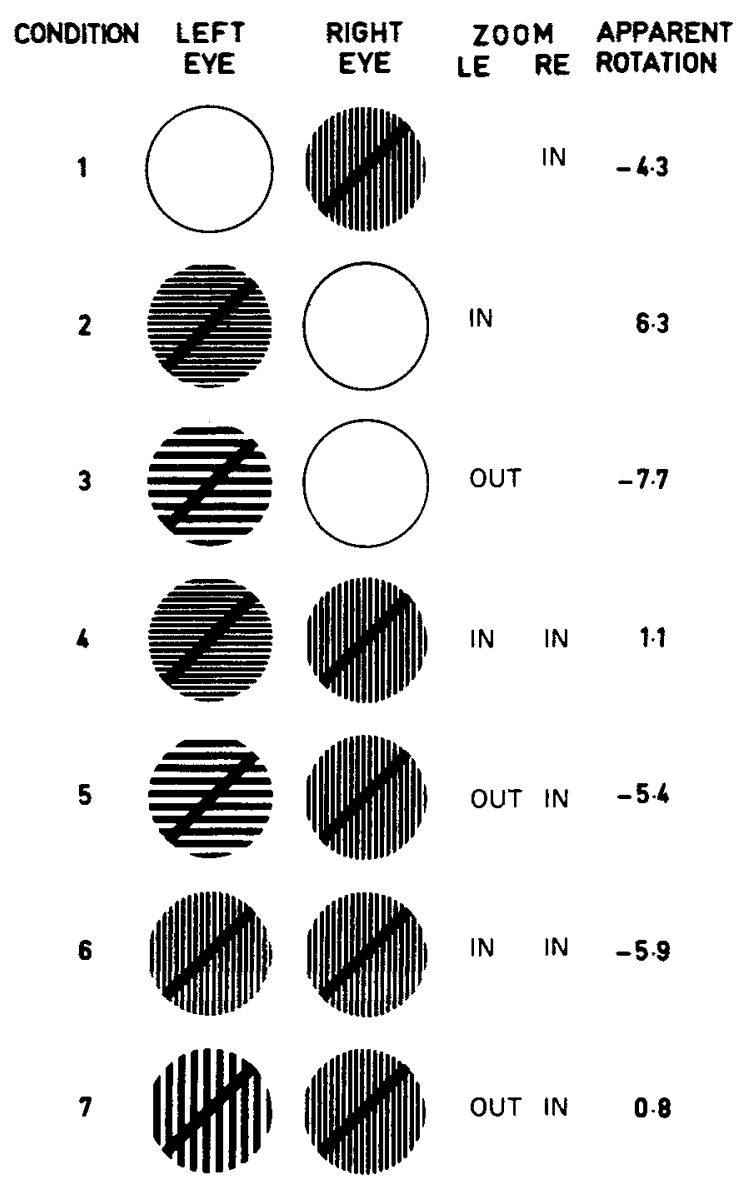

Figure 4. The initial stimulus displays and the zoom phases for Experiment 3. The mean degrees of apparent rotation are also given for each condition (negative values denote counterclockwise rotation).

three monocular conditions gave rise to a mean reported rotation in the expected direction (see Swanston \& Wade, 1981). Thus, a counterclockwise perceived rotation occurred with zoom-in on a vertical background or zoomout on a horizontal background. In Condition 4 , the backgrounds were vertical and horizontal, and both received zoom-in. The rotation of about $1^{\circ}$ was close to the difference between the same conditions viewed monocularly (Conditions 1 and 2). The same principle applied to the other dichoptic conditions. In Condition 5, the left eye viewed a horizontal background zoomed out, and the right eye, a vertical background zoomed in. This produced a net rotation of around $5.4^{\circ}$ counterclockwise, intermediate between the values for Conditions 1 and 3 . When both eyes viewed the same pattern of stimulation in Condition 6 , the perceived rotation was about $1.5^{\circ}$ more than in the equivalent monocular case (Condition 1). In Condition 7, as in Condition 4, the two fields had opposite effects due to the different directions of zooming, and the net rotation was close to zero.

In each dichoptic condition, the perceived rotation was therefore predictable from the effects of the same fields viewed independently and monocularly. Whether the background orientations or zoom directions in each eye produced the same or different directions of apparent rotation, the binocular outcome approximated the mean of the two.

\section{DISCUSSION}

The evidence from three experiments lends little support to the suggestion that binocular interaction takes place in this type of induced motion. The simple dichoptic presentation of the inducing and target figures (Experiment 1) abolished the apparent rotation of the line against the expanding background. However, binocular rivalry between the line and the contralateral background provided a potential confounding effect, which two further experiments were designed to avoid. A clear dichoptic apparent rotation was produced in Experiment 2, but this could be accounted for by the additive effects of the component parts, which were measured independently. When the split fields induced oppositely directed rotations of the line, the dichoptic effect was abolished. In Experiment 3, an oblique line was presented to both eyes, but the background could differ either in orientation or zoom phase. Again, the results in the dichoptic conditions could be predicted from the monocular components, which were measured independently.

It seems unlikely that these results would have been influenced by pursuit eye movements. The background inducing fields expanded or contracted radially, giving a perceived motion symmetrically about a central vertical axis (see Swanston \& Wade, 1981). Tracking of any background line would reduce the retinal velocity of the inducing field in one direction and increase it correspondingly in the opposite half of the display. However, the symmetry of the background movement makes it unlikely that any systematic bias of this sort would have occurred.

The displays used in these experiments have one feature in common with those of Over and Lovegrove (1973) and Day and Dickinson (1977), in which no dichoptic interaction was observed; they were presented within a stationary circular framework. Here the static surround was provided by the circular masks in front of the monitors, which defined the limits of the displays in the darkened laboratory and acted as fusional aids. When the whole frame containing a grating is displaced laterally, movement can be induced dichoptically (Day \& Dickinson, 1977). In those cases in which dot-and-frame-induced motion was shown dichoptically (Bassili \& Farber, 1977; Day \& Dickinson, 1977), lateral displacement of the whole frame occurred. These results suggest that induced movement effects can be subdivided according to the degree to which they persist during dichoptic presentation. If the distinction suggested above is sustained, this might in turn furnish useful evidence for interpreting induced movement effects generally. That is, object-relative motion might be extracted visually at a very early stage, whereas any subject-relative displacements would require a higher level of integration. 


\section{REFERENCES}

ANstis, S., \& DunCan, K. (1983). Separate motion aftereffects from each eye and from both eyes. Vision Research, 23, 161-169.

ANSTIS, S. M., \& ReINHARDT-RutLand, A. H. (1976). Interactions between motion aftereffects and induced movement. Vision Research, 16, 1391-1394.

Bassili, J. N., \& FARBer, J. M. (1977). Experiments on the locus of induced motion. Perception \& Psychophysics, 21, 157-161.

Brosgole, L., Cristal, R. M., \& Carpenter, O. (1968). The role of eye movements in the perception of visually induced motion. Perception \& Psychophysics, 3, 166-168.

DAY, R. H., Dickinson, R. G. (1977). Absence of color-selectivity in Duncker-type induced movement. Perception \& Psychophysics, 22, 313-320.

DUNCKER, K. (1929). Über induzierte Bewegung. Psychologische Forschung, 12, 180-209.

EHRENSTEIN, W. (1925). Versuche über die Beziehungen zwischen Bewegung und Gestaltwahrnehmung. Zeitschrift für Psychologie, 96, 305-352.

Gogel, W. C., \& GriffiN, B. W. (1982). Spatial induction of illusory motion. Perception, 11, 187-199.

Gogel, W. C., \& KosLow, M. (1972). The adjacency principle and induced movement. Perception \& Psychophysics, 11, 309-314.

KaUfman, L. (1974). Sight and mind. New York: Oxford University Press.

LEVI, D. M., \& Schor, C. M. (1984). Spatial and velocity tuning of processes underlying induced motion. Vision Research, 24, 1189-1196.

Moulden, B. (1980). After-effects and the integration of patterns of neural activity within a channel. Philosophical Transactions of the Royal Society (London), Series B, 290, 39-55.

OVER, R., \& LoVegrove, W. (1973). Color-selectivity in simultaneous motion contrast. Perception \& Psychophysics, 14, 445-448.

Schulman, P. H. (1979). Eye movements do not cause induced motion. Perception \& Psychophysics, 26, 381-383.

Swanston, M. T., \& WADE, N. J. (1981). Apparent rotation of a line superimposed upon radially expanding and contracting backgrounds. Perception, 10, 265-271.

WADE, N. J. (1980). The influence of colour and contour rivalry on the magnitude of the tilt illusion. Vision Research, 20, 229-233.

WADE, N. J., \& SwaNSTON, M. T. (1984a). Illusions of size change in dynamic displays. Perception \& Psychophysics, 35, 268-290.

Wade, N. J., \& Swanston, M. T. (1984b). Perceptual transformations in moving displays. In L. Spillman \& B. Wooten (Eds.), Sensory experience, adaptation and perception (pp. 547-560). London: Erlbaum.

Wade, N. J., Swanston, M. T., ONo, H., \& Wenderoth, P. (1983). Factors influencing the apparent rotation of a line on radially transformed displays. Perception, 12, 313-322.

WALLACH, H. (1959). The perception of motion. Scientific American, 201, 56-60.

Wallach, H., BaCon, J., \& Schulman, P. (1978). Adaptation in motion perception: Alteration of induced motion. Perception \& Psychophysics, 24, 509-514.

(Manuscript received July 23, 1984; revision accepted for publication March 18, 1985.)

\section{ERRATUM}

Cormack, R., \& Fox, R. The computation of retinal disparity. Perception \& Psychophysics, 1985, 37, 176-178-Please note the following corrections: (1) In the legend of Figure 2, "tangent" should be replaced by "target." (2) Equation 8 should read as follows:

$$
r=\{\operatorname{ATN}[(J+A f) / D f]+\operatorname{ATN}[(J-A f) / D f]\}-\{\operatorname{ATN}[(J+A t) / D t]+\operatorname{ATN}[(J-A t) / D t]\} .
$$

(3) In Equations 11 and 12, the mathematical symbol to the right of "radians" should be an equals sign (=). (4) In the second to the last line from the bottom in the first column on page 178, "asymmetric" should read "symmetric." 